\title{
Avaliação genética do tamanho de leitegada em suínos das raças Landrace e Large White
}

\author{
[Genetic evaluation of litter size in swine of Landrace and Large White breeds] \\ S.F. Lázaro ${ }^{1}$, V.P.S. Felipe ${ }^{1}$, F.M. Gonçalves ${ }^{1}$, T.L. Passafaro ${ }^{1}$, \\ M.A. Silva ${ }^{2}$ \\ ${ }^{1}$ Alunos de pós-graduação - Escola de Veterinária - UFMG - Belo Horizonte, MG \\ ${ }^{2}$ Bolsista CNPq - Escola de Veterinária - UFMG - Belo Horizonte, MG
}

\begin{abstract}
RESUMO
Objetivou-se com este estudo estimar parâmetros genéticos para o número total de leitões nascidos (NTLN), número de leitões nascidos vivos (NLNV) e número de leitões vivos aos cinco dias de idade (NLV5) com modelos de regressão aleatória e averiguar melhor modelagem da variância residual na avaliação das trajetórias genéticas do tamanho da leitegada de fêmeas Landrace e Large White. Os dados utilizados foram provenientes de uma granja de melhoramento genético de suínos e continham 2.388 observações de fêmeas Landrace e 2.325 de Large White. Os modelos de melhor ajuste para o NTLN e NLV5 foram os que consideraram a variância residual homogênea e, para NLNV, o modelo com quatro classes de variâncias residuais foi o mais adequado (BIC). Para Landrace, o efeito materno não foi significativo. O modelo que incluiu o efeito materno e quatro classes de variância residual foi o que apresentou melhor ajuste para NTLN na raça Large White, sendo os modelos sem efeito materno e com variância residual homogênea os mais adequados para NLNV e NLV5. As herdabilidades estimadas variaram de baixas a altas $(0,08-0,34 ; 0,04-0,29$ e $0,05-0,21$ na raça Landrace e 0,16-0,30; 0,10-0,37 e 0,09-0,32 na Large White, para NTLN, NLNV e NLV5, respectivamente). A alta correlação de posto entre os valores genéticos do NLNV e NLV5 sugere que não há necessidades do controle do NLV5 nesse programa de melhoramento genético. Maiores ganhos podem ser obtidos pela seleção no NLNV de fêmeas primíparas, em função da diminuição do intervalo de gerações.
\end{abstract}

Palavras-chave: leitões, correlação, herdabilidade, modelo

\begin{abstract}
This study aimed to estimate genetic parameters for total number of piglets born (NTLN), number of piglets born alive (NLNV) and number of piglets alive at five days of age (NLV5) using random regression models and to evaluate the best way for modelling the residual variance in the description of the genetic trajectories of litter size in Landrace and Large White breeds. The data came from a swine breed improvement program, and a total of 2388 and 2325 litter size records of Landrace and Large White, respectively were used in the analyses. The models considering homogenous residual variance showed the best goodness of fit for NTLN and NLV5 and the model with four classes of residual variances was most appropriate for NLNV (BIC). In the Landrace breed the maternal effect was not significant. The model including maternal effect and four classes of residual variance adequately described NTLN of Large White breed and the models without maternal effect and with homogeneous residual variance were most appropriate to describe NLNV and NLV5. The estimated heritability for NTLN, NLNV and NLV5 ranged from low to high (0.08-0.34, 0.04-0.29 and 0.05-0.21 in Landrace breed and 0.16-0.30, 0.10-0.37, 0.09-0.32 in Large White breed.). The magnitude of the rank correlations between breeding values of NLNV and NLV5 suggests that the recording of NLV5 is not necessary in this breed improvement program. High genetic gains can be obtained by selecting NLNV of primiparous females, due to the reduction in the generation interval.
\end{abstract}

Keywords: piglet, correlation, heritability, model

Recebido em 20 de março de 2013

Aceito em 25 de junho de 2014

E-mail: sirlenelazaro@yahoo.com.br 


\section{INTRODUÇÃO}

A raça de suínos Large White foi criada em meados de 1700, na Inglaterra, por meio do cruzamento entre animais de uma raça chinesa de Canton e a raça Yorkshire. Desde então foi estabelecida como a raça mais utilizada em todos os países produtores de suínos (Taylor et al., 2005). A raça Landrace, por sua vez, foi desenvolvida na Dinamarca a partir do cruzamento do suíno nativo com a raça Large White. Esse cruzamento foi melhorado durante anos com controle rígido do governo. Sua criação visava à produção de toucinho e adaptabilidade ao sistema intensivo, além de se destacar pela prolificidade. Assim, em virtude das características promissoras de determinadas raças, o melhoramento animal tem por finalidade aperfeiçoar a produção (Lourenço et al., 2008).

A obtenção de material genético de qualidade para essas raças requer programas de melhoramento bem fundamentados, que forneçam animais com valores genéticos acurados e precisos. A genética de suínos no Brasil é uma das mais avançadas do mundo, sendo a alta qualidade e especificidade dos reprodutores (machos e fêmeas) utilizados nas granjas comerciais, um dos segmentos de maior evolução (Holanda et al., 2005; Rohenkohl, 2009). Os descendentes desses animais de alto valor genético para características produtivas e reprodutivas geram retorno econômico para os criadores e atendem às exigências do consumidor.

De acordo com Rydhmer (2000), o tamanho da leitegada é a característica reprodutiva mais importante economicamente. Além disso, é considerada em vários programas de melhoramento em função das provas convincentes de que tem respondido com êxito à seleção (Sorensen et al., 2000; Noguera et al., 2002), fato demonstrado quando se usam essas raças especializadas.

As características longitudinais, ou seja, aquelas medidas várias vezes durante a vida do animal, como o tamanho de leitegada, podem ser analisadas por meio de modelos de repetibilidade, que consideram todas as medidas da característica como sendo a mesma característica (Pereira et al., 2000), ou por modelos multicaracterísticas, que consideram cada medida da mesma variável como uma característica diferente (Gressler et al., 2000). No entanto, alguns pesquisadores reconhecem que, para esse tipo de característica, os modelos de regressão aleatória são mais apropriados, por acomodarem todos os registros de cada característica que mudam gradualmente ao longo da trajetória de produção, sem qualquer tipo de ajuste (Mercadante et al., 2002).

Portanto, objetivou-se, com a realização desta pesquisa, estimar parâmetros genéticos para o número total de leitões nascidos, número de leitões nascidos vivos e número de leitões vivos aos cinco dias de idade com modelos de regressão aleatória utilizando polinômios de Legendre de diferentes ordens e verificar qual melhor modelo para avaliar as trajetórias genéticas do tamanho da leitegada de fêmeas Landrace e Large White em função da ordem de parição.

\section{MATERIAL E MÉTODOS}

Os dados utilizados foram provenientes de uma granja de melhoramento genético de suínos do Estado de Minas Gerais. O banco de dados era composto por número total de leitões nascidos (NTLN), número de leitões nascidos vivos (NLNV) e número de leitões vivos aos cinco dias (NLV5) de animais puros das raças Landrace e Large White. $\mathrm{O}$ arquivo da raça Landrace era composto por dados de 929 fêmeas com 2.388 partos, ocorridos de 2005 a 2010, e arquivo de pedigree contendo 1.108 animais. Já o arquivo da raça Large White era constituído por informações de 860 fêmeas com 2.325 partos, ocorridos de 2005 a 2010, e arquivo de pedigree com 1.038 animais.

As análises de consistência e a edição do banco de dados foram realizadas por meio de ferramentas do EXCEL. Dados como ordem de parto duplicada, animais com identificação menor que a de seus pais e época de parição menor do que a época de nascimento foram eliminados.

Os tamanhos das leitegadas foram analisados por meio de modelo animal em regressão aleatória. As regressões fixas e aleatórias foram representadas por funções contínuas, cujas ordens de parições foram descritas em termos de polinômios ortogonais de Legendre com a 
padronização da parição $j\left(p_{j}\right.$, da escala original) para o intervalo entre -1 e $1\left(p_{j}^{*}\right)$ (Lukovic et al., 2003):

$$
i p_{j}^{*}=\frac{2\left(p_{j}-p_{\min }\right)}{p_{\max }-p_{\min }}-1
$$

em que: $p_{\min }$ e $p_{\max }$ representam a primeira e a última parição do intervalo original, respectivamente. Os modelos ajustados por funções de diferentes ordens $\left(2^{\mathrm{a}}, 3^{\mathrm{a}}, 4^{\mathrm{a}}, 5^{\mathrm{a}}\right.$ e $6^{\mathrm{a}}$ ordem) para os efeitos aleatórios considerando o efeito genético materno (modelo 2) ou não (modelo 1), para as três características avaliadas, podem ser representados como segue:

Modelo 1: $y=X \beta+Z_{1} \alpha+Z_{3} \rho+e$

Modelo 2: $y=X \beta+Z_{1} \alpha+Z_{2} m+Z_{3} \rho+e$

em que: $y$ representa o vetor com o tamanho da leitegada dos animais; $X$, a matriz de incidência de efeitos fixos de grupo contemporâneo (mês e

$$
E=\left(\begin{array}{c}
y \\
a \\
m \\
\rho \\
e
\end{array}\right)=\left[\begin{array}{c}
X \beta \\
0 \\
0 \\
0 \\
0
\end{array}\right] e \quad V\left[\begin{array}{c}
\alpha \\
m \\
\rho \\
e
\end{array}\right]=\left[\begin{array}{cccc}
K_{a} \otimes A & \sigma_{K_{a} K_{m}} & 0 & 0 \\
\sigma_{K_{a} K_{m}} & K_{m} \otimes A & 0 & 0 \\
0 & 0 & K_{p} \otimes I & 0 \\
0 & 0 & 0 & R
\end{array}\right]
$$

$K_{a}, K_{m}$ e $\mathrm{K}_{p}$ são, respectivamente, as matrizes de (co)variância entre os coeficientes de regressão aleatória do efeito genético aditivo, materno e de ambiente permanente direto; $A$ é a matriz dos numeradores dos coeficientes de parentesco, de Wright, entre os indivíduos; $I$ é a matriz identidade; $\otimes$ é o produto direto; $R$ é matriz diagonal de variância residual.

Os componentes de (co)variância entre coeficientes de regressão aleatórios, para obtenção das estimativas de herdabilidades, correlações (genéticas e de ambiente permanente de animal), e valores genéticos das reprodutrizes, para o modelo selecionado com base em critérios de comparação de modelos, foram obtidos pelo método da Máxima Verossimilhança Restrita (REML), utilizando-se o programa Wombat (Meyer, 2007).

As análises foram realizadas considerando-se alternativamente homogeneidade e heterogeneidade de variância residual. Na definição das variâncias residuais, foram consideradas as seguintes ano de parição) e idade da porca ao parto; $\beta$, o vetor com as soluções para os efeitos fixos e coeficientes de regressão que descrevem a trajetória média da população; $Z_{l}$, a matriz de incidência com os coeficientes dos polinômios utilizados para modelar os efeitos genéticos aditivos diretos; $\alpha$, o vetor com as soluções para os coeficientes de regressão aleatória dos efeitos genéticos aditivos diretos; $Z_{2}$, a matriz de incidência com os coeficientes dos polinômios utilizados para modelar os efeitos genéticos maternos; $m$, o vetor com as soluções para os coeficientes de regressão aleatória dos efeitos genéticos maternos; $Z_{3}$, a matriz de incidência com os coeficientes dos polinômios utilizados para modelar os efeitos de ambiente permanente direto; $\rho$, o vetor com as soluções para os coeficientes de regressão aleatória dos efeitos de ambiente permanente direto e $e$, o vetor com os resíduos. E assume-se;

classes de ordem de parição: uma classe de variância residual - homogeneidade de variância residual -; duas classes de variância residual ordem de parição 1 a 3 e 4 a 6 -; três classes de variância residual - ordem de parição 1 a 2, 3 a 4 e 5 a 6 -; quatro classes de variância residual ordem de parição $1,2,3$ a 4 e 5 a 6 -; cinco classes de variância residual - ordem de parição $1,2,3,4$ e 5 a 6 -; seis classes de variância residual - ordem de parição 1, 2, 3, 4, 5 e 6 .

Os modelos, com diferentes graus polinomiais e estruturas de variância residual foram comparados, inicialmente, pela mudança no logaritmo da função de máxima verossimilhança $(\operatorname{LogL})$, por meio do teste da razão de verossimilhança (LRT). A estatística LRT foi obtida pela seguinte expressão: $\mathrm{LRT}_{\mathrm{ij}}=2 \log \mathrm{L}_{\mathrm{i}}$ - $2 \log L_{j}$, em que $\log L_{i}$ é o máximo da função de verossimilhança para o modelo completo $i$, e $\log L_{j}$ é o máximo da função de verossimilhança para o modelo reduzido $j$. E também foram comparados pelo critério de informação bayesiano de Schwartz (BIC), que permite 
comparação entre os modelos não hierárquicos e impõe penalidades de acordo com o número de parâmetro a ser estimado. $\mathrm{O}$ modelo com menor valor é considerado o de melhor ajuste e, segundo Huisman et al. (2002), o BIC é o mais rígido e pode ser definido como:

$$
B I C=-2 * \log L+p^{*} \log (N-r(X))
$$

em que: $p$ é o número de parâmetros estimados; $N$, o número de observação; $r(X)$, o posto da matriz de incidência dos efeitos fixos do modelo; e $\log L$, o logaritmo da função de máxima verossimilhança restrita (Wolfinger, 1993).

\section{RESULTADOS E DISCUSSÃO}

As informações dos modelos utilizados constam na Tabela 1 , incluindo os modelos que mais se ajustaram aos dados, de acordo com o critério de escolha de modelos BIC. Para as três características avaliadas, verificaram-se menores valores de logaritmo de verossimilhança ( $\log \mathrm{L})$ nos modelos com homogeneidade de variância residual.

Tabela 1. Número de parâmetros (Np), logaritmo de verossimilhança (Log L), valores de LRT e BIC para os modelos ajustados, considerando o efeito materno nos modelos

\begin{tabular}{|c|c|c|c|c|c|c|c|}
\hline Raça & Modelo & $\mathrm{ka}$ & $\mathrm{kp}$ & $\mathrm{e}$ & $\mathrm{Np}$ & - $\log \mathrm{L}$ & $\mathrm{BIC}$ \\
\hline \multirow{14}{*}{ Landrace } & \multicolumn{7}{|c|}{ Sem efeito materno } \\
\hline & \multirow{3}{*}{$\mathrm{NTLN}^{\mathrm{a}}$} & 2 & 2 & 1 & 7 & $-3928,0$ & $\mathbf{7 8 7 9 , 7 0}$ \\
\hline & & 4 & 4 & 3 & 23 & $-3917,4$ & 7912,36 \\
\hline & & 2 & 2 & 4 & 10 & $-3883,8$ & 7801,39 \\
\hline & \multirow[t]{2}{*}{$\mathrm{NLNV}^{\mathrm{b}}$} & 5 & 5 & 3 & 33 & $-3869,6$ & 7850,54 \\
\hline & & 2 & 2 & 1 & 7 & $-3888,9$ & 7801,35 \\
\hline & $\mathrm{NLV}^{\mathrm{c}}$ & 5 & 5 & 3 & 33 & $-3866,6$ & 7844,46 \\
\hline & \multicolumn{7}{|c|}{ Efeito materno } \\
\hline & \multirow{3}{*}{ NTLN } & 2 & 2 & 1 & 10 & $-3927,1$ & 7887,97 \\
\hline & & 5 & 5 & 2 & 47 & $-3910,9$ & 7980,17 \\
\hline & & 2 & 2 & 1 & 10 & $-3761,9$ & 7557,49 \\
\hline & \multirow[t]{2}{*}{ NLNV } & 6 & 6 & 2 & 65 & $-3736,7$ & 7692,15 \\
\hline & & 2 & 2 & 5 & 14 & $-3878,6$ & 7804,46 \\
\hline & NLV5 & 5 & 5 & 2 & 47 & $-3865,7$ & 7889,71 \\
\hline \multirow{14}{*}{ Large White } & \multicolumn{7}{|c|}{ Sem efeito materno } \\
\hline & \multirow{3}{*}{ NTLN } & 3 & 3 & 2 & 14 & $-4025,6$ & $\mathbf{8 0 9 8 , 3 7}$ \\
\hline & & 5 & 5 & 4 & 34 & $-4013,0$ & 8139,67 \\
\hline & & 2 & 2 & 1 & 7 & $-3786,9$ & 7597,36 \\
\hline & \multirow[t]{2}{*}{ NLNV } & 3 & 3 & 6 & 18 & $-3779,8$ & 7620,21 \\
\hline & & 2 & 2 & 1 & 7 & $-3851,0$ & 7725,54 \\
\hline & NLV5 & 4 & 4 & 6 & 26 & $-3836,2$ & 7759,75 \\
\hline & \multicolumn{7}{|c|}{ Efeito materno } \\
\hline & \multirow{3}{*}{ NTLN } & 2 & 2 & 4 & 13 & $-4032,2$ & 8108,12 \\
\hline & & 6 & 6 & 4 & 67 & $-4001,8$ & 8228,21 \\
\hline & & 2 & 2 & 1 & 10 & $-3786,7$ & 7606,99 \\
\hline & \multirow[t]{2}{*}{ NLNV } & 5 & 5 & 1 & 46 & $-3776,1$ & 7706,66 \\
\hline & & 2 & 2 & 1 & 10 & $-3850,5$ & 7734,63 \\
\hline & NLV5 & 6 & 6 & 1 & 64 & $-3826,4$ & 7867,42 \\
\hline
\end{tabular}

${ }^{a}$ Número total de leitões nascidos; ${ }^{b}$ Número de leitões nascidos vivos; ${ }^{c}$ Número de leitões vivos aos 5 dias; $k_{a}$ : ordem do polinômio para o efeito genético; $k_{p}$ : ordem do polinômio para o efeito de ambiente permanente; $e$ : número de classes residuais consideradas.

Os modelos que proporcionaram melhor ajuste para o NTLN e NLV5 na raça Landrace foram os que consideraram a variância residual homogênea e não incluíram o efeito materno 
(BIC), assim como para o NLNV e NLV5 em animais Large White.

De acordo Cavalcante-Neto et al. (2011), as variações ao longo das curvas de produção em suínos são atribuídas a diversos fatores, como estádio fisiológico, condições do parto, duração do período seco, etc., que não são facilmente incorporados aos modelos de análise, em razão de não existirem informações sobre esses fatores. Com isso, essas variações são redirecionadas ao resíduo, sendo, portanto, necessário atribuir diferentes estruturas para o resíduo, o que mostra a importância de se considerar a heterogeneidade da variância residual por meio de modelos de regressão aleatória.

Neste contexto, para as características NTLN e NLNV, em Large White e Landrace, respectivamente, os modelos que consideraram a heterogeneidade de variância residual com quatro classes foram os mais adequados (BIC) e, ainda, para os animais Large White, o efeito materno foi incluído no modelo (NTLN). Por fim, verificou-se que o ajuste do efeito materno não foi significativo para nenhuma das três características estudadas na raça Landrace.

Observou-se leve declínio nas estimativas de variâncias genéticas entre o primeiro e o segundo parto, com aumentos a partir do terceiro parto, para a raça Landrace (Tab. 2). Em contrapartida, para a raça Large White, verificou-se que essas foram crescentes com o aumento do número de partos. Comportamento diferente entre as duas raças também foi verificado para a variância de ambiente permanente que, em geral, foi decrescente ao longo das parições para Landrace e crescente para Large White, o que pode ser atribuído às diferenças genéticas entre as duas raças.

As estimativas de variância genética foram mais elevadas nas últimas parições para os dois grupos genéticos (Tab. 2). Esse resultado está associado ao período de menor número de registros de leitegadas, em função da seleção das matrizes, que é realizada nesse programa de melhoramento por meio da combinação de dados de desempenho (ganho de peso diário, espessura de toucinho e porcentagem de carne magra), e do Índice de Produtividade de Fêmea (IPF - composto pelo NLV5 e peso da leitegada à desmama). Assim, as fêmeas deixam normalmente de duas a três leitegadas, podendo ocorrer dificuldades no ajuste dos modelos, resultando em estimativas mais elevadas nas últimas parições.

A herdabilidade, em geral, aumentou de acordo com a ordem de parto para todas as características avaliadas (Tab. 2). Esse fato sugere a existência de diferentes bases genéticas atuando no tamanho da leitegada ao longo da vida reprodutiva das porcas (Barbosa et al., 2008). Entretanto, as estimativas de herdabilidade apresentaram grande variação ao longo dos partos $(0,04$ a 0,34 - Landrace, e 0,09 a 0,37 - Large White). Assim como, para as variâncias genética e de ambiente permanente, o comportamento das herdabilidades também foi diferente em ambas as raças, com leve declínio entre o primeiro e o segundo parto e aumento a partir do terceiro parto para Landrace e crescente em todo o período para Large White, sendo maiores os valores observados nas últimas parições (Tab. 2).

Irgang et al. (1994) também encontraram grandes variações nas estimativas de herdabilidade $(0,021-0,283)$ em suínos Landrace, Large White e Duroc. Os valores observados nas últimas parições por esses autores se assemelham aos encontrados nas últimas parições deste trabalho; no entanto, eles utilizaram apenas os três primeiros partos para obterem as estimativas. Por outro lado, Lukovic et al. (2004), ao estimarem parâmetros genéticos em animais das raças Landrace e Large White por intermédio de modelos de regressão aleatória, com seis parições, encontraram valores de herdabilidade que pouco variaram $(0,10-0,13)$.

Lima et al. (2007), ao estudarem o comportamento de porcas primíparas e multíparas na fase reprodutiva, verificaram que as fêmeas multíparas estão mais adaptadas às condições gerais do ambiente e apresentam menor tempo na realização de determinado comportamento e índices zootécnicos melhores do que as primíparas, o que pode explicar a diminuição nas estimativas de herdabilidade do primeiro para o segundo parto nas fêmeas Landrace. 
Tabela 2. Estimativas das variâncias genética $\left(\sigma_{\mathrm{a}}^{2}\right)$, de ambiente permanente $\left(\sigma_{\mathrm{pem}}^{2}\right)$, residual $\left(\sigma_{\mathrm{e}}^{2}\right) \mathrm{e}$ fenotípica $\left(\sigma_{\mathrm{p}}^{2}\right)$ e herdabilidades $\left(\mathrm{h}^{2}\right)$, obtidas por modelos de regressão aleatória para os modelos que melhor se ajustaram aos dados para cada característica e raça

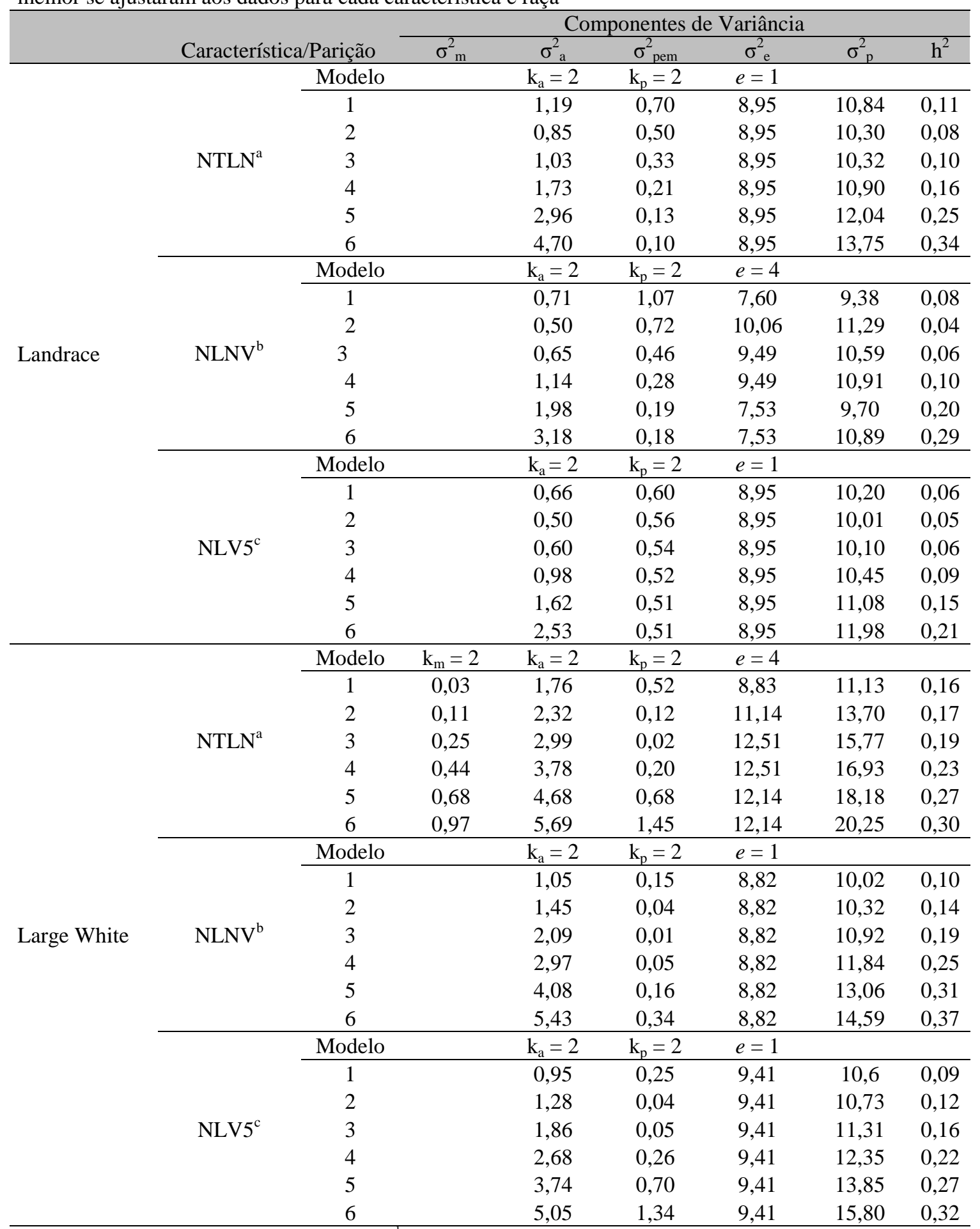

NTLN $^{\mathrm{a}}$ : Número total de leitões nascidos; NLNV ${ }^{\mathrm{b}}$ : Número de leitões nascidos vivos; $\mathrm{NLV}^{\mathrm{c}}$ : Número de leitões vivos aos cinco dias; $k_{a}$ : ordem do polinômio para o efeito genético; $k_{p}$ : ordem do polinômio para o efeito de ambiente permanente; $k_{m}$ : ordem do polinômio para o efeito genético materno; $e$ : número de classes residuais consideradas. 
Portanto, estimativas obtidas em diferentes rebanhos, com composições genéticas variadas e criados em ambientes distintos, não são totalmente comparáveis, uma vez que fatores como população, raça, condições de criação, sistemas de seleção, entre outros, podem determinar diferentes estimativas de herdabilidade para uma mesma característica.

As correlações genéticas para número de leitões nascidos vivos e vivos aos cinco dias (Tab. 3) foram maiores entre parições adjacentes para $\operatorname{NLNV}(0,83$ a 0,99 e 0,96 a 0,99 para Landrace e Large White, respectivamente) e para NLV5
(0,81 a 0,99 e 0,95 a 0,99 para Landrace e Large White, respectivamente), e decresceram à medida que o intervalo entre as parições aumentou.

As estimativas das correlações de ambiente permanente, assim como das correlações genéticas, foram maiores entre parições adjacentes e decresceram à medida que $\mathrm{o}$ intervalo de partos aumentou (-0,171 a 0,992 e 0,971 a 0,997 para NLNV, em Landrace e Large White, respectivamente) $(-0,052$ a 0,992 e 0,905 a 0,999 para NLV5, em Landrace e Large White, respectivamente).

Tabela 3. Estimativas de correlações de efeito genético aditivo direto (diagonal superior) e de efeito de ambiente permanente (diagonal inferior) para número de leitões nascidos vivos e para número de leitões nascidos vivos (NLNV e NLV5) nas diversas parições, obtidas por modelo de regressão aleatória

\begin{tabular}{|c|c|c|c|c|c|c|c|}
\hline \multicolumn{2}{|l|}{ Raça } & \multicolumn{6}{|c|}{ NLNV } \\
\hline \multirow{14}{*}{ Landrace } & Parição & 1 & 2 & 3 & 4 & 5 & 6 \\
\hline & 1 & & 0,996 & 0,974 & 0,888 & 0,605 & 0,057 \\
\hline & 2 & 0,880 & & 0,990 & 0,925 & 0,673 & 0,145 \\
\hline & 3 & 0,506 & 0,855 & & 0,969 & 0,770 & 0,283 \\
\hline & 4 & 0,162 & 0,611 & 0,933 & & 0,903 & 0,510 \\
\hline & 5 & $-0,047$ & 0,432 & 0,837 & 0,978 & & 0,830 \\
\hline & 6 & $-0,171$ & 0,316 & 0,763 & 0,944 & 0,992 & \\
\hline & Parição & \multicolumn{6}{|c|}{ NLV5 } \\
\hline & 1 & & 0,998 & 0,992 & 0,981 & 0,966 & 0,945 \\
\hline & 2 & 0,893 & & 0,998 & 0,991 & 0,980 & 0,963 \\
\hline & 3 & 0,577 & 0,883 & & 0,998 & 0,991 & 0,979 \\
\hline & 4 & 0,272 & 0,676 & 0,943 & & 0,998 & 0,99 \\
\hline & 5 & 0,069 & 0,511 & 0,855 & 0,979 & & 0,998 \\
\hline & 6 & $-0,058$ & 0,398 & 0,782 & 0,945 & 0,992 & \\
\hline \multirow{14}{*}{ Large White } & Parição & \multicolumn{6}{|c|}{ NLNV } \\
\hline & 1 & & 0,965 & 0,901 & 0,837 & 0,783 & 0,738 \\
\hline & 2 & 0,971 & & 0,983 & 0,951 & 0,918 & 0,889 \\
\hline & 3 & 0,185 & 0,416 & & 0,992 & 0,975 & 0,958 \\
\hline & 4 & $-0,738$ & $-0,554$ & 0,526 & & 0,996 & 0,987 \\
\hline & 5 & $-0,866$ & $-0,720$ & 0,330 & 0,976 & & 0,998 \\
\hline & 6 & $-0,904$ & $-0,775$ & 0,252 & 0,955 & 0,997 & \\
\hline & Parição & \multicolumn{6}{|c|}{ NLV5 } \\
\hline & 1 & & 0,956 & 0,873 & 0,792 & 0,725 & 0,672 \\
\hline & 2 & 0,905 & & 0,978 & 0,937 & 0,896 & 0,860 \\
\hline & 3 & $-0,609$ & $-0,214$ & & 0,989 & 0,969 & 0,948 \\
\hline & 4 & $-0,866$ & $-0,572$ & 0,924 & & 0,995 & 0,984 \\
\hline & 5 & $-0,912$ & $-0,651$ & 0,881 & 0,995 & & 0,997 \\
\hline & 6 & $-0,929$ & $-0,684$ & 0,859 & 0,990 & 0,999 & \\
\hline
\end{tabular}

As estimativas de correlações genéticas entre as várias parições foram altas e positivas, à exceção das correlações entre a quinta e a sexta parição com as demais, o que sugere que a seleção não deve ser realizada tardiamente. Até a quarta parição, todas as correlações genéticas entre os partos foram maiores do que 0,80 e, segundo Robertson (1959), esse valor seria o limiar para a característica começar a perder 
importância biológica; no entanto, a maior correlação genética foi obtida entre o $1^{\circ}$ e $2^{\circ}$ parto $(0,996)$, ou seja, a seleção de fêmeas primíparas poderia ser mais eficiente.

A seleção na primeira parição, nessa situação, proporcionaria ganhos por meio da resposta correlacionada, uma vez que as correlações genéticas nesse período ( $1^{\mathrm{a}}$ a $4^{\mathrm{a}}$ parição) foram todas maiores que 0,80 , indicando que a maioria dos genes que atua em uma parição atua nas demais e no mesmo sentido; portanto, os melhores animais nas primeiras parições também podem ser melhores nas demais parições (Robertson, 1959; Cavalcante-Neto et al., 2011), o que diminuiria o intervalo de gerações do plantel, gerando ganhos genéticos para a população.

Em rebanhos nos quais os suínos possuam maior valor de mercado, pode ser interessante o aproveitamento das fêmeas de reposição por maior número de partos. Dessa forma, as matrizes podem permanecer no rebanho até a quarta parição, uma vez que as estimativas de correlação genética neste estudo foram altas da primeira à quarta parição. Contudo, em rebanhos núcleos, deve-se considerar alta intensidade de seleção (i), maior correlação entre o agregado genotípico e o índice de seleção $\left(\mathrm{r}_{\mathrm{HI}}\right)$ e menor intervalo de gerações, para maximização do progresso genético. Nesse caso, a seleção de fêmeas primíparas resultaria em maior ganho genético decorrente principalmente do menor intervalo de gerações e também pela alta correlação genética para o número de leitões nos partos imediatamente subsequentes.

Por fim, por meio da correlação de Spearman (correlação de posto) entre os valores genéticos do NLNV e NLV5, período que considera a capacidade de criação da fêmea, e menor mortalidade dos leitões (Su et al., 2007), foram obtidos valores, tanto para o primeiro parto quanto para o segundo, próximos da unidade $(0,99)$, sugerindo que o registro do NLV5 poderia ser dispensado.

\section{CONCLUSÕES}

Os efeitos da heterogeneidade e da homogeneidade da variância residual devem ser testados na modelagem das características do tamanho da leitegada em suínos. As altas correlações genéticas indicam que o tamanho da leitegada ao nascer é tão eficiente quanto o tamanho da leitegada aos cinco dias de idade, podendo-se dispensar o controle zootécnico do último em programas de melhoramento genético de suínos destas populações (Landrace e Large White). Maiores ganhos genéticos podem ser obtidos pela seleção no tamanho da leitegada ao nascer de fêmeas primíparas, em função da diminuição do intervalo de gerações, e não da redução na acurácia dos valores genéticos.

\section{REFERÊNCIAS}

BARBOSA, L.; LOPES, P.S.; REGAZZI, A.J. et al. Estimação de parâmetros genéticos em tamanho de leitegada de suínos utilizando análises de características múltiplas. Rev. Bras. Zootec., v.37, p.1947-1952, 2008.

CAVALCANTE-NETO, A.; THOLON, P.; LUI, J.F. et al. Modelos de regressão aleatória com diferentes estruturas de variância residual para descrever o tamanho da leitegada. Rev. Cienc. Agro., v.42, p.1043-1050, 2011.

GRESSLER, S.L.; BERGMANN, J.A.G.; PEREIRA, C.S. et al. Estudo das associações genéticas entre perímetro escrotal e características reprodutivas de fêmeas nelore. Rev. Bras. Zootec., v.29, p. 427-437, 2000.

HOLANDA, M.C.R.; BARBOSA, S.B.P.; SAMPAIO, I.B.M. et al. Tamanho da leitegada e pesos médios, ao nascer e aos 21 dias de idade, de leitões da raça Large White. Arq. Bras. Med, Vet. Zootec., v.57, p.539-544, 2005.

HUISMAN, A.E.; VEERKAMP, R.F.; VAN ARENDONK, J.A.M. Genetic parameters for various random regression models to describe the weight data of pigs. J. Anim. Sci., v.80, p.575-582, 2002.

IRGANG, R.; FAVERO, J.A.; KENNEDY, B.W. Genetic parameters for litter size of different parities in Duroc, Landrace, and Large White sows. J. Anim. Sci., v.72, p.2237- 2246, 1994.

LIMA, K.R.S.; PEDREIRA, H.A.; JESUS, M.L.C. et al. Estudo do comportamento de porcas primiparas e multiparas na fase reprodutiva. In: BRASILEIRO DE ZOOTECNIA, 17, 2007, Londrina-PR. Anais... Londrina-PR: UEL, 2007 Disponível em:http://www.abz.org.br/publicaçõestecnicas/anais-zootec/artigos-cientificos/reproduçãomelhoramento-animal/3040-Estudo-comportamentoporcas-primparas-multparas-fase-reprodutiva.html. Acessado em: 10/11/2012. 
LOURENÇO, F.F.; DIONELLO, N.J.L.; MEDEIROS, G.C.R. et al. Estudos genéticos sobre a leitegada em suínos da raça Landrace criados no Rio Grande do Sul. R. Bras. Zootec., v.37, p.1601-1606, 2008.

LUKOVIC, Z.; MALOVRH, S.; GORJANC, G. et al. Genetic parameters for number of piglets born alive using a random regression model. Agric. Conspec. Sci., v.68, p.105-108, 2003.

LUKOVIC, Z.; MALOVRH, S.; GORJANC, G. et al. A random regression model in analysis of litter size in pigs. South African J. Anim. Sci., v.34, p.241248, 2004.

MERCADANTE, M.E.Z.; PACKER, I.U.; RAZOOK, A.G. et al. Dias ao Parto de Fêmeas Nelore de um Experimento de Seleção para Crescimento. II - Modelo de Regressão Aleatória. Rev. Bras. Zootec., v.31, p.1726-1733, 2002.

MEYER, K. WOMBAT - A tool for mixed model analyses in quantitative genetics by restricted maximum likelihood (REML). J. Zhejiang Univ.SCI. B, v.8, p.815-821, 2007.

NOGUERA, J.L.; VARONA, L.; BABOT, D. et al. Multivariate analysis of litter size for multiple parities with production traits in pigs: II. Response to selection for litter size and correlated response to production traits. J. Anim. Sci. v.80, p.2548-2555, 2002.

PEREIRA, E.; ELER, J.P.; STERMAN, J.B.F. Correlação Genética Entre Perímetro Escrotal e Algumas Características Reprodutivas na Raça Nelore. Rev. Bras. Zootec., v.29, p.1676-1683, 2000.
ROBERTSON, A. The Sampling Variance of the Genetic Correlation Coefficient. Biomet., v.15, p.469-485, 1959.

ROHENKOHL, J.E. Dinâmica tecnológica e ambiente seletivo em genética de suínos. Rev. Bras. Inov., v.2, p.403-435, 2009.

RYDHMER, L. Genetics of sow reproduction, including puberty, oestrus, pregnancy, farrowing and lactation. Livest. Prod. Sci., v.66, p.1-12, 2000.

SORENSEN, D.; VERNERSEN, A.; ANDERSEN, S. Bayesian analysis of response to selection: A case study using litter size in Danish Yorkshire pigs. Genetics, v.156, p.283-295, 2000.

SU, G.; LUND, M.S.; SORENSEN, D. Selection for litter size at day five to improve litter size at weaning and piglet survival rate. J. Anim. Sci., v.85, p.1385-1392, 2007.

TAYLOR, G.; ROESE, G.; HERMESCH, S. Breeds of pigs-Landrace. NSW DPI - Primefacts, 63: Profitable \& Sustainnable Primary Industries, 2005. Disponível em http://http://www.dpi.nsw.gov.au/_data/assets/pdf_ file/0008/45557/Breeds_of_pigs-Landrace__Primefact_63-final.pdf. Acessado em: 19/04/2014.

WOLFINGER, R. Covariance structure selection in general mixed models. Commun. Stat. Simulat., v.22, p.1079-1106, 1993. 